\title{
Rare Tumors of the Neuroectodermal Origin: Report of Five Cases
}

\author{
Vladimir Bauer, Zorica Aleric, Ervin Jancic, Tatjana Bujas
}

\begin{abstract}
We present a case series of five patients who were diagnosed in our institution with rare tumors of neuroectodermal origin. They were discovered within 1 year, by two otorhinolaryngologists at the county hospital which covers the area of about $1,00,000$ inhabitants. Neuroectodermal tumors of the head and neck are rare pathologies, with extremely varied clinical behavior, treatment and prognosis. The lack of symptom specificity, the low incidence of these tumors and the difficulties associated with histopathology are main reasons for delayed diagnosis and treatment. We point out that rare tumors, if considered individually, have a low incidence, some even negligible, but the pathology detected hundreds of types which increases the likelihood that we will encounter some of them in our practice. Second, the practice in medicine does not always follow the statistical and mathematical probabilities but should take into account less possible occurrences.
\end{abstract}

Keywords: Malignant peripheral nerve sheat tumor, Neuroblastoma, Neuroectodermal tumors, Neuroendocrine carcinoma, Rare tumors of the head and neck, Schwannoma.

How to cite this article: Bauer V, Aleric $Z$, J ancic E, Bujas T. Rare Tumors of the Neuroectodermal Origin: Report of Five Cases. Int J Head Neck Surg 2012;3(2):108-111.

\section{Source of support $\mathrm{Nil}$}

\section{Conflict of interest: None}

\section{INTRODUCTION}

Neuroectodermal tumors arise from cells derived from the primitive neuroectoderm including Schwann cells, neuronal cells of the peripheral nervous system (enteric, parasympathetic, sympathoadrenal and sensory neurons), pigment cells (melanocytes), endocrine cells, neuroglial cells, parenchymal cells of pineal gland, neuronal cells of the retina and cells forming connective tissue of the face and neck. ${ }^{1}$ Tumors exhibiting neuroectodermal differentiation occur throughout the body, and the diverse tissues of the head and neck give rise to a wide assortment of these neoplasms. ${ }^{2}$ All of them are uncommon in the head and neck region.

Some of them are very rare, have little or no chance to be seen during someone's career and are not to be thought about in the differential diagnosis. Due to their rarity, the clinical and pathologic characteristics of these neoplasms are not adequately understood and no reliable treatment has been established. In this study, a retrospective review analysis was performed on 5 patients who were diagnosed in our institution with rare tumors of neuroectodermal origin.
They were discovered within 1 year, by two otorhinolaryngologists at the county hospital which covers the area of about $1,00,000$ inhabitants.

\section{CASE REPORTS}

\section{Case 1}

Patient 1 is a 67-year-old men presented with 3 weeks history of pain, impairment of vision and protrusion of the right eye. A fter being examined by the ophthal mologist, the tumor formation was found in the right nasal cavum. On endoscopy, a red, necrotic, polypoid mass with a tendency to bleed was seen. Multislice computed tomographic (M SCT) scans (Figs $1 A$ and $B$ ) showed a breakthrough in
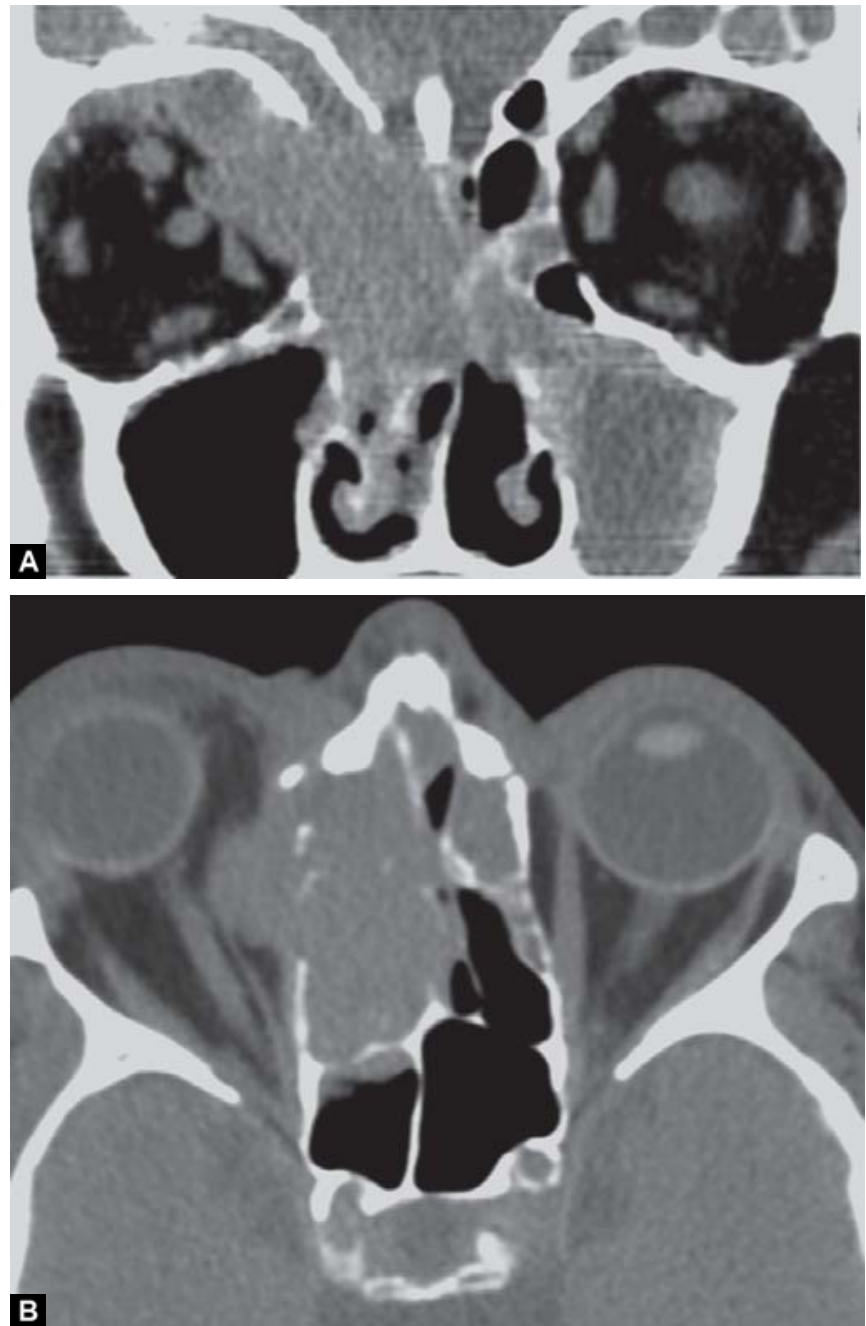

Figs $1 \mathrm{~A}$ and $\mathrm{B}$ : MSCT scans in coronal view $(\mathrm{A})$ and axial view (B) showing extension of the tumor in frontal and maxillary sinus, orbit and endocranially 
etmoide, frontal sinus, orbit and endocranially. A fter failure by endonasal approach, an adequate sample for analysis was extracted through the frontal sinus. A biopsy of the mass was interpreted as sinonasal neuroendocrine carcinoma. Because of the extent of the process and generally the poor condition of the patient, the treatment was symptomatic.

\section{Case 2}

Patient 2 is a 62-year-old male smoker. A fast growing formation on the forehead was a first sign of illness in our patient. Fine-needle aspiration cytology (FNA C) from soft tissue mass revealed mesenchymal tumor. It was removed and sent to pathohistological (PH) verification, which revealed small cell neuroendocrine carcinoma. Within a month multiple metastases on the lungs, liver, bones and brain were detected. Also a recurrent tumor developed on the frontal region with concomitant invasion to the skull and frontal lobe (Figs $2 A$ and B). Neurosurgical tumor resection with partial removal of the bone was performed at clinical hospital. He died 4 months after the first surgery. The opinions w ere divided on was it a primary tumor of the skin or lungs so the skin changes that we treated were actually rare lung tumor metastases.

\section{Case 3}

Patient 3 is a 36-year-old man presented as a typical right sided nasal polyposis. CT scans confirmed that

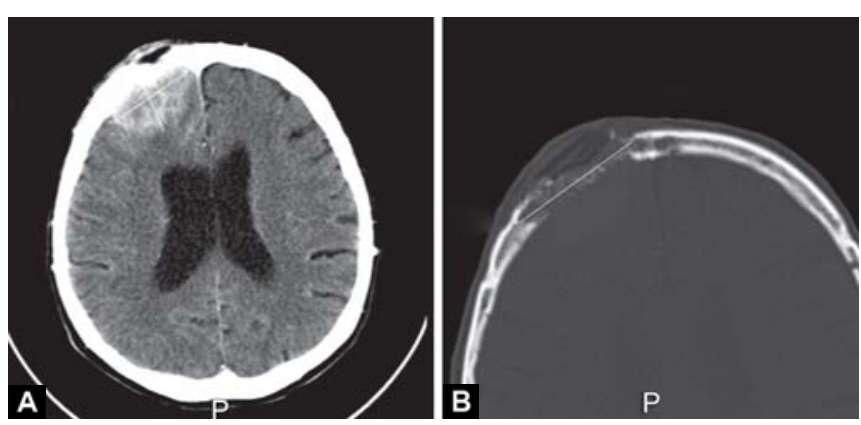

Figs 2A and B: MSCT scans showing intracranially extension of the tumor $(A)$ and destruction of the frontal bone $(B)$

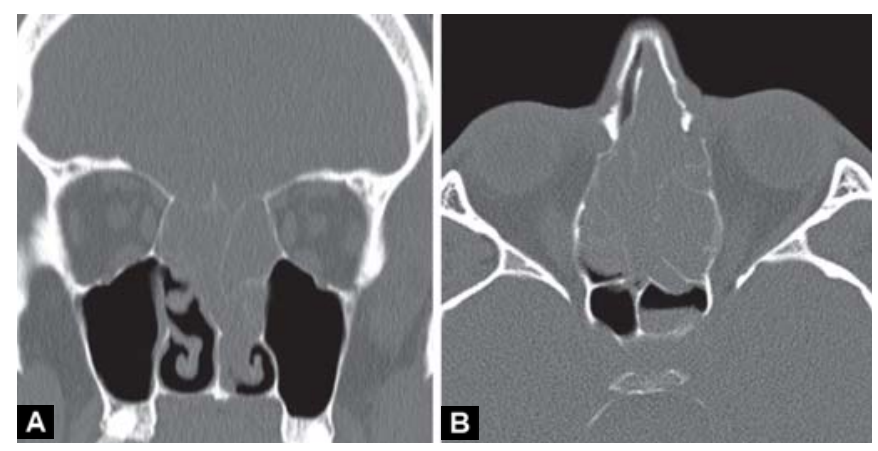

Figs 3A and B: Coronal $(A)$ and axial $(B)$ view of the esthesioneuroblastoma
(Figs $3 A$ and B). Nasal obstruction, frontal headache and secretion was the most important symptoms. The surgery was interrupted due to anusually heavy bleeding. A biopsy of the mass was interpreted as olfactory neuroblastoma. Extended surgery was performed at clinical hospital. PH findings show ed us that sometimes what appears as benign polyp can be a malignant tumor. Now, 18 months after surgery he is without any evidence of recurrent disease.

\section{Case 4}

Patient 4 is a 49-year-old man originally complained of nonspecific problems, which included fatigue and poor general condition. Signs of H orner's syndrome were noticed in the somatic status of his left eye, however, without visual disturbances. A spherical formation of rubbery consistency was palpated in the left supraclavicular area. The M SCT described the solitary expansive process in the base of the neck on the left side, spreading into the mediastinum to the level of the third thoracic vertebra (Figs $4 \mathrm{~A}$ and B). FNAC from soft tissue mass revealed mesenchimal tumor. The surgery was performed through the neck and tumor described as $6 \times 5 \times 3 \mathrm{~cm}$ in size was removed. $\mathrm{A} \mathrm{PH}$ findings of the mass was interpreted as schwannoma. Regard to the Horner's syndrome signs and intraoperative findings, we conclude that tumor originated from sympathetic chain.

\section{Case 5}

Patient 5 is a 76-year-old men presented with 1 month history of pain, swelling and progressive, rapid growth of left-sided cervical mass. FNAC was indeterminate. The patient underwent biopsy of the mass which reveled malignant peripheral neural sheet tumor. On first physical examination, a $9 \times 7 \mathrm{~cm}$ large, hard and fixed mass was palpated, only 2 weeks after the large diameter was $15 \mathrm{~cm}$ mostly occupying the entire left side of the neck. M SCT scans of the head and neck showed a expansive, solid, polycyclic formation (Figs $5 A$ and $B$ ). B ecause of extension and extremely rapid growth treatment was symptomatic. Tracheostomy was performed. Since, the patient denied
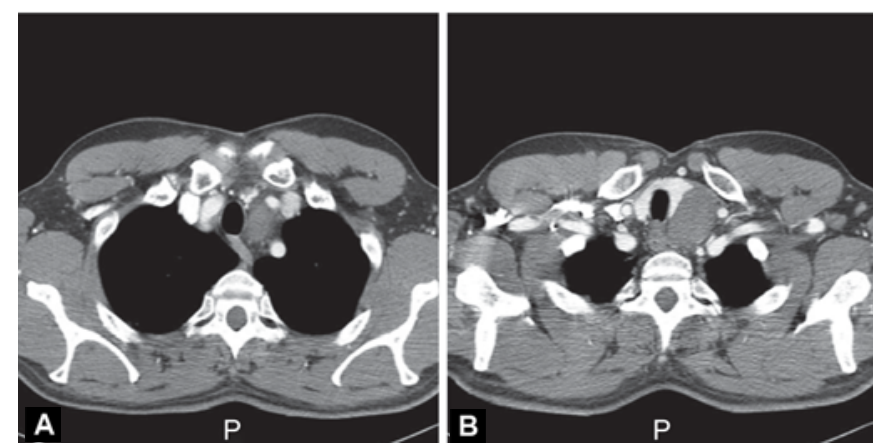

Figs 4A and B: MSCT scans showing intrathoracic extension of the schwannoma (A) and cervicomediastinal extension (B) 


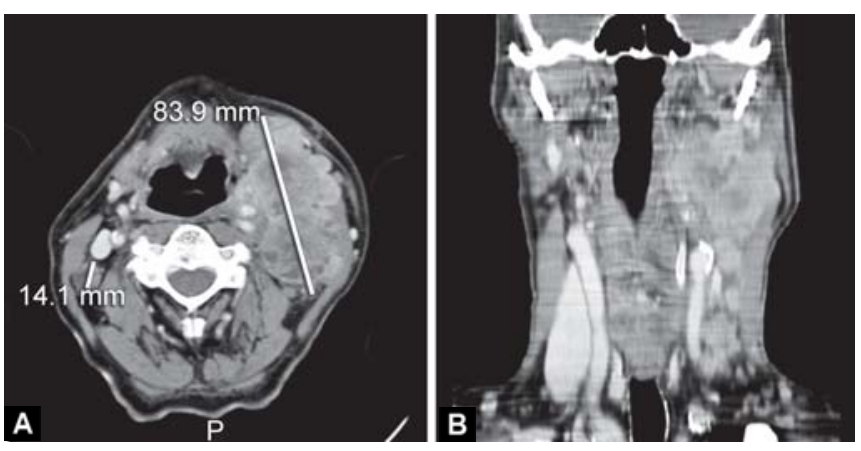

Figs 5A and B: Axial (A) and coronal $(B)$ view of the huge cervical malignant peripheral nerve sheat tumor

previous benign pathology that may have been likened to be neurofibromatosis, a de novo origin may be thought of for the present case.

\section{DISCUSSION}

Neuroendocrine carcinoma (poorly differentiated) has the incidence of $0.5 / 1,00,000$ after adding up all the localizations in the body. ${ }^{3}$ The $2005 \mathrm{~W}$ orld Health Organization classification of head and neck tumors categorizes these neoplasms as typical carcinoid tumor, atypical carcinoid tumor, small-cell carcinoma neuroendocrine type and neuroendocrine carcinoma 'not otherwise specified'. ${ }^{1}$

Schwannomas of cervical sympathetic chain origin are very rare. A bout 50 cases are described in the literature, all concerning the entire paravertebral sympathetic chain, usually in the cervical region. ${ }^{4}$

Olfactory neuroblastoma or esthesioneuroblastoma is an uncommon neuroendocrine malignancy which accounts for approximately $3 \%$ of endonasal neoplasms and $0.8 \%$ of all human cancers. It is a malignant neoplasm that is highly aggressive and has a high recurrence rate with distant metastases, particularly in intracranial invasion. ${ }^{5}$

The incidence of malignant peripheral nerve sheath tumor is approximately $0.001 \%$. M alignant peripheral nerve sheath tumors (MPNST) is also known as malignant schwannoma, neurofibrosarcoma, malignant neurilemmoma and neurogenic sarcoma. ${ }^{6} \mathrm{M}$ ost cases are developed sporadically $(50 \%), 31 \%$ arise from a malignant transformation of schwannoma and 19\% from a neurofibroma. This tumor is usually found in the lower extremities and only 10 to $12 \%$ of all lesions occur in the head and neck region, which all makes it a rare entity. ${ }^{7}$

Despite the use of modern diagnostic devices, such as multislice computerized tomography, magnetic resonance imaging, angiography, ultrasound controlled FNA C-all this tumors still represents a diagnostic problem. There are no specific radiological features, however, imaging is useful in assessing of the extent. Combination of M SCT and M R I technique is favorable. Like in our case, FNAC does not contribute significantly to the differential diagnosis of neuroectodermal tumors.

It may be difficult or sometimes impossible to establish a definitive diagnosis. Differentiation from other neoplasms may be unrealible, when only small, mechanically distorted, biopsy specimens are available. More different biopsy specimens could be necessary. In all our cases immunohistochemical investigation was performed to confirm the diagnosis.

Despite the substantial progress in treatment modalities available in the present era, the wide spreading nature and rapid growth of this tumors has a strong hold in determining the prognosis. Three of our presented patients died because of advanced and untreatable stage of disease without any therapy except symptomatic one. Early detection of this aggressive species of tumors may help reduce morbidity.

$W$ ith this presentation of rare tumors of neuroectodermal origin in the head and neck area, we want to draw the attention to two facts. First, rare tumors, if considered individually, have a low incidence, some even negligible, but the development of pathology as a science detected hundreds of types which increases the likelihood that we will encounter some of them in our practice. Second, the practice in medicine does not always follow the statistical and mathematical probabilities but should take into account less possible occurrences.

\section{REFERENCES}

1. Perez-Ordonez B. N euroendocrine tumors. In: Barnes L, Eveson J W, R eichart P, Sidransky D (Eds). W orld Health Organization classification of tumours. Pathology and genetics. Head and neck tumours. Lyon: AIRC Press 2005:26-27.

2. W enig B, Dulguerov P, K apadia S, Prasad M , F anburg-Smith J, Thompson L. Neuroectodermal tumors. In W orld Health Organization classification of tumours pathology and genetics of head and neck tumours. Barnes L, Eveson J, Reichart P, Sidransky D (Eds). Lyon: IA RC Press 2005:66-70.

3. Rare cancers list - M arch 2011 Surveillance of rare cancers in Europe. A vailible from: http://www.rarecare.eu/rarecancers/ rarecancers.asp

4. Jain S, Houseknecht K, Rojiani AM, Setzer M, V rionis FD. $M$ anagement of nerve sheath tumors arising in the sympathetic chain. Cancer Control 2008;15(4):352-57.

5. Thompson LD. O Ifactory neuroblastoma. Head and N eck Pathol 2009;3:252-59.

6. Ziadi A, Saliba I. M alignant peripheral nerve sheath tumor of intracranial nerve: A case series review. A uris Nasus Larynx 20100 ct;37(5):539-45.

7. Patil K, M ahima VG, A mbika L. M alignant peripheral nerve sheath tumour: An elusive diagnosis. Indian J Dent Res 2007; 18:19-22. 


\section{ABOUT THE AUTHORS}

\section{Vladimir Bauer (Corresponding Author)}

Department of Otorhinolaryngology, General Hospital Karlovac Karlovac, Croatia, e-mail: vbauermail@yahoo.com

\section{Zorica Aleric}

Department of Otorhinolaryngology, General Hospital Karlovac Karlovac, Croatia

\section{Ervin Jancic}

Department of Neurology, General Hospital Karlovac, Karlovac Croatia

\section{Tatjana Bujas}

Department of Pathology, General Hospital Karlovac, Karlovac Croatia 